\title{
Optimizing hydroxyl airglow retrievals from long-slit astronomical spectroscopic observations
}

\author{
Christoph Franzen ${ }^{1,2, *}$, Robert Edward Hibbins ${ }^{1,2}$, Patrick Joseph Espy ${ }^{1,2}$, and Anlaug Amanda Djupvik ${ }^{3}$ \\ ${ }^{1}$ Norwegian University of Science and Technology (NTNU), 7491 Trondheim, Norway \\ ${ }^{2}$ Birkeland Centre for Space Science (BCSS), Birkeland, Norway \\ ${ }^{3}$ Nordic Optical Telescope, 38700 Santa Cruz De La Palma, Spain \\ * Invited contribution by Christoph Franzen, recipient of the EGU Atmospheric Sciences Outstanding Student Poster and \\ PICO Award 2016.
}

Correspondence to: Christoph Franzen (christoph.franzen@ntnu.no)

Received: 3 February 2017 - Discussion started: 22 March 2017

Revised: 27 June 2017 - Accepted: 11 July 2017 - Published: 25 August 2017

\begin{abstract}
Astronomical spectroscopic observations from ground-based telescopes contain background emission lines from the terrestrial atmosphere's airglow. In the near infrared, this background is composed mainly of emission from Meinel bands of hydroxyl $(\mathrm{OH})$, which is produced in highly excited vibrational states by reduction of ozone near $90 \mathrm{~km}$. This emission contains a wealth of information on the chemical and dynamical state of the Earth's atmosphere. However, observation strategies and data reduction processes are usually optimized to minimize the influence of these features on the astronomical spectrum. Here we discuss a measurement technique to optimize the extraction of the $\mathrm{OH}$ airglow signal itself from routine $\mathrm{J}$-, $\mathrm{H}$-, and $\mathrm{K}$-band long-slit astronomical spectroscopic observations. As an example, we use data recorded from a point-source observation by the Nordic Optical Telescope's intermediate-resolution spectrograph, which has a spatial resolution of approximately $100 \mathrm{~m}$ at the airglow layer. Emission spectra from the $\mathrm{OH}$ vibrational manifold from $v^{\prime}=9$ down to $v^{\prime}=3$, with signal-to-noise ratios up to 280 , have been extracted from $10.8 \mathrm{~s}$ integrations. Rotational temperatures representative of the background atmospheric temperature near $90 \mathrm{~km}$, the mesosphere and lower thermosphere region, can be fitted to the $\mathrm{OH}$ rotational lines with an accuracy of around $0.7 \mathrm{~K}$. Using this measurement and analysis technique, we derive a rotational temperature distribution with $v^{\prime}$ that agrees with atmospheric model conditions and the preponderance of previous work. We discuss the derived rotational temperatures from the different vibrational bands and highlight the potential for both the archived and future observations, which are at unprecedented spatial
\end{abstract}

and temporal resolutions, to contribute toward the resolution of long-standing problems in atmospheric physics.

\section{Introduction}

\subsection{OH airglow}

The atmospheric region between 85 and $90 \mathrm{~km}$ represents a transition zone that lies between the thermosphere, where collisions are rare, and the collision-dominated, wellmixed mesosphere. This mesosphere and lower thermosphere (MLT) region is highly variable both chemically and dynamically (Smith, 2004, 2012). It is driven from above by diurnal, seasonal, and long-term changes in solar insolation and from below by tides, planetary waves, and upwardpropagating gravity waves.

Photochemical reactions in the MLT play a key role in the vertical distribution of energy. The major loss for mesopause ozone is its reduction to molecular oxygen via

$\mathrm{H}+\mathrm{O}_{3} \rightarrow \mathrm{OH}^{*}+\mathrm{O}_{2}$.

This loss is balanced by the major source of the ozone, the combination of molecular and atomic oxygen via a mediator M:

$\mathrm{O}+\mathrm{O}_{2}+\mathrm{M} \rightarrow \mathrm{O}_{3}+\mathrm{M}$

where the atomic oxygen is formed by the dissociation of molecular oxygen by solar radiation (Espy and Stegman, 
2002; Sigernes et al., 2003). Reaction (R1) is highly exothermic $(>3 \mathrm{eV})$, leading to production of vibrational levels from $v^{\prime}=7$ to $v^{\prime}=9$ of the $\mathrm{OH}$ product. Deactivation of these high vibrational states primarily occurs through photo-emission in the Meinel bands (Meinel, 1950), resulting in the bright $\mathrm{OH}$ airglow, localized in a thick ( $\sim 8 \mathrm{~km}$ thick) layer near $90 \mathrm{~km}$, that can be observed in the visible and infrared. Collisions of each long-lived vibrational excited state $\left(v^{\prime}\right)$ with the surrounding gas effectively thermalize the lower, closely spaced rotational states into a Boltzmann distribution (Pendleton et al., 1993). Hence, moderate-resolution spectroscopic measurements of the relative population of the rotational levels of individual $\mathrm{OH}$ vibrational bands can be used to remotely sense the temperature of the mesopause region. Furthermore, the relative intensity of the individual vibrational bands can be used to estimate the relative populations of the $v^{\prime}$ states. Comprehensive reviews on ground-based observations of $\mathrm{OH}$ and their applications to mesopause chemistry can be found in von Zahn et al. (1987), Yee et al. (1997), and Smith et al. (2010).

However, achieving high temporal resolution has often only been possible at low spatial resolution (and vice versa). In this paper we use $\mathrm{J}-, \mathrm{H}-$, and $\mathrm{K}$-band long-slit observations of an astronomical point source made by the Nordic Optical Telescope near-infrared camera and spectrograph (NOTCam). This intermediate-resolution spectrometer was used to obtain high-quality, very high spatial $(<100 \mathrm{~m})$ resolution observations with short integration times $(\sim 10 \mathrm{~s})$. We demonstrate how to extract and optimize $\mathrm{OH}$ atmospheric spectral data from these astronomical observations and discuss the quality and validity of the derived data over the range of vibrational bands. Finally, we consider some problems of atmospheric physics that can be addressed with these new data.

\subsection{Instrumentation}

Founded in 1984 and located in La Palma, Spain $\left(17^{\circ} 53^{\prime} \mathrm{W}\right.$, $28^{\circ} 45^{\prime} \mathrm{N}$ ), the Nordic Optical Telescope (NOT) has a primary mirror with a diameter of $2560 \mathrm{~mm}$ (Djupvik and Andersen, 2010). Its near-infrared camera and spectrograph, NOTCam (Abbott et al., 2000), has been used for imaging since June 2001 and spectroscopy since August 2003. The detector is a $1024 \times 1024$ pixel Rockwell Science Center HgCdTe Astronomical Wide Area Infrared Imaging (HAWAII) array. The low-resolution mode ( $R=\lambda / \Delta \lambda \sim 2100)$ is sufficient to resolve the individual rotational lines of an $\mathrm{OH}$ vibrational level. The dispersing element is an echelle grism used together with broadband filters to sort the orders. In low-resolution spectroscopic mode the slit employed has a width of $128 \mu \mathrm{m}$, corresponding to approximately $0.6 \operatorname{arcsec}$ on the sky, and a slit length of 4 arcmin. At an approximate $\mathrm{OH}$ layer altitude of $87 \mathrm{~km}$ (Baker and Stair Jr., 1988), 4 arcmin corresponds to around $100 \mathrm{~m}$ on the sky. The detector has a dead time of about $10 \mathrm{~s}$ due to reading out processes after each integration. Further details on NOTCam spectroscopy can be found in Telting (2016), and a description of the NOTCam in Djupvik (2016).

During point-source astronomical observations, measurements in the $\mathrm{J}, \mathrm{H}$, and $\mathrm{K}$ bands (1.165-1.328, 1.484-1.780, and $2.038-2.363 \mathrm{~nm}$, respectively) are typically taken. Although each of these wavelength bands is observed individually, atmospheric $\mathrm{OH}$ vibrational band transitions $(7,4)$ and $(8,5)$ are simultaneously observed in the $\mathrm{J}$ band; the $(3,1),(4,2),(5,3)$, and a part of the $(6,4)$ in the $\mathrm{H}$ band; and the $(8,6)$ and $(9,7)$ in the $\mathrm{K}$ band (Meinel, 1950). The $(9,7)$ band is of particular interest as it represents the highest vibrational level populated by Reaction (R1).

\section{Data reduction}

To demonstrate the data reduction procedures required to optimize the $\mathrm{OH}$ signal from routine astronomical observations, a single $\mathrm{H}$-band spectroscopic exposure toward the star $21 \mathrm{Vir}$ (spectral type B9V, $H=5.64 \mathrm{mag}$ ) with an exposure time of $10.8 \mathrm{~s}$ was used. This exposure time was chosen as an example as it is the shortest exposure time available in the archive, although the methods presented here were developed and optimized for a variety of conditions with integration times up to $600 \mathrm{~s}$, which is the longest integration time available in the archive.

The H-band image presented here was taken in good astronomical observing conditions at an air mass of 1.516 at 05:50 UT on 19 February 2013, together with dark frames and flat fields recorded with a halogen lamp on the same night. The J- and K-band images presented in Sect. 3.2 were recorded toward the same object with the same integration time within $5 \mathrm{~min}$ of the $\mathrm{H}$-band observation. The raw image frame is reproduced in Fig. 1a. The vertical bright band in Fig. 1a, located slightly to the left on the detector, is the H-band spectrum of the star. The weaker, nearly horizontal lines that curve upward are the atmospheric $\mathrm{OH}$ lines from the $(3,1),(4,2),(5,3)$, and (part of the) $(6,4)$ vibrational bands, listed from top to bottom in the image frame.

For the initial data reduction steps standard Image Reduction and Analysis Facility (IRAF; Tody, 1993) astronomical procedures were followed. This entailed removing bad pixels (zero valued or cold) that are known and stable, and dark frames were used to create a pixel mask to remove hot pixels on the detector. The intensity of the $\mathrm{OH}$ lines is well within the linear range of the array, so non-linearity effects could be ignored. The dark current was removed using dark images taken toward the closed dome before observations began. Wavelength-dependent variations in transmission and detector response were corrected by flat fielding using short integrations of a halogen lamp with a known colour temperature situated at the upper end of the optical path. 

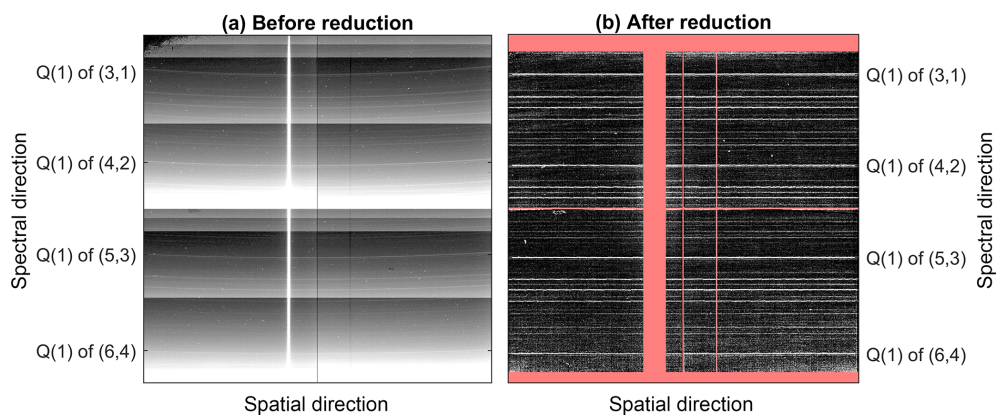

Figure 1. (a) The array in its raw form. The bright star is visible as a vertical band slightly to the left of centre. The curved OH lines are visible in the background. (b) The array after cleaning and flat fielding, with the star removed and the $\mathrm{OH}$ lines straightened. The unusable parts of the array are blanked out in red, and the $\mathrm{OH}$ lines can be seen clearly.

The stellar spectrum is much brighter than the $\mathrm{OH}$ lines and has to be removed. A linear mask, 60 pixels wide in the spatial direction and centred on the star's horizontal position, was applied to the detector frame. Sixty pixels removed the stellar influence while maintaining as much $\mathrm{OH}$ data as possible. The remaining $\mathrm{OH}$ lines do not appear as straight lines on the array due to the intersection of the array detector with the telescope's diffraction optics. Instead they are parabolic, with a curvature that varies linearly with spectral position. To straighten them for integration, a line's spatial and spectral pixels $\left(x, y^{\prime}\right)$ were mapped to a coordinate system where the line appears at the same spectral location, $y$, along the entire spatial extent, $x$, using the function $y=y^{\prime}+\left(x-p_{1}\right)^{2} \cdot p_{2}$. The detector is aligned such that the central position of the parabola, $p_{1}$, is constant at column number 465 , and the curvature, $p_{2}$, is given by $p_{2}=3 \times 10^{-5}+5.3 \times 10^{-8} \cdot y^{\prime}$. The result after processing is shown in Fig. $1 \mathrm{~b}$.

Given this transformation, the $\mathrm{OH}$ lines could then be integrated in the spatial dimension, $x$, for a given value of wavelength, $y$, with the standard deviation used to estimate each line's uncertainty, to form a high signal-to-noise spectrum (after accounting for the pixels masked out as described above). Gaussian functions with a full width at half maximum of $0.42,0.73$, and $0.87 \mathrm{~nm}$ were found to fit the line shape of the resulting $\mathrm{OH}$ lines in the centres of the $\mathrm{J}, \mathrm{H}$, and $\mathrm{K}$ bands, respectively, to within the noise present in the data. The fitting however is performed in units of pixels rather than nanometres. The line width is constant in pixel units for each band separately at 2.27 pixels.

Since the wavelengths of the $\mathrm{OH}$ lines are known (Rothman et al., 2013), and the lines are easy to identify, the abundant lines themselves were used for wavelength calibration. The brightest lines of the $\mathrm{Q}$ branch and the six brightest Pbranch lines of each transition were used for the calibration of each filter band. For the H-band example presented here, this led to a calibration based on 22 pixel-wavelength pairs (only the main Q-branch lines were used for the $(6,4)$ transition). The wavelength calibration has only small non-linear contributions.

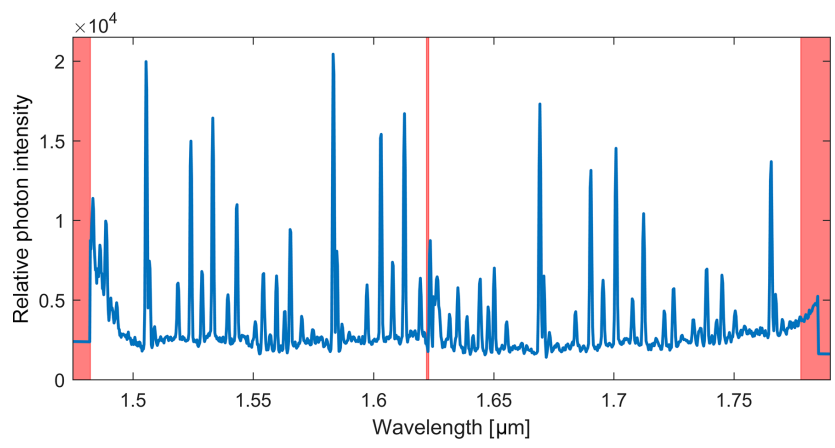

Figure 2. H-band spectrum of atmospheric $\mathrm{OH}$ extracted from the data frame presented in Fig. 1. Red shading highlights parts of the spectrum not used in the subsequent rotational temperature fitting.

\section{Results}

\subsection{H-band data}

In Fig. 2 the resulting H-band spectrum is shown based on the total integration time of $10.8 \mathrm{~s}$. The spectrum has been corrected using a relative spectral radiance calibration derived from the flat-field source. However, an absolute calibration could be achieved using observations of a standard star of known intensity. The absolute calibration is however not needed for this work as we are only interested in the hydroxyl temperatures. The flat fielding distorts the edges of the spectrum slightly as the filter transmission nears zero in the red highlighted regions in the figure. At the long-wavelength end, the Q and R branches from the $(6,4)$ transition can be seen. The other three resolved bands belong to the $(3,1)$, $(4,2)$, and $(5,3)$ transitions. The signal-to-noise ratio is 280 for the Q-branch lines and around 200 for the P-branch lines for this $10.8 \mathrm{~s}$ exposure. Longer integration times increase these ratios further.

As can be seen in Fig. 2, the background is not completely flat but has low-frequency variations that span wavelength ranges greater than the $\mathrm{OH}$ line widths. Since these background variations will influence the relative intensities of the 
individual rotational lines, they impact the fitted $\mathrm{OH}$ rotational temperature since it is strongly dependent on the relative heights of the lines. For this reason a seventh-order Butterworth infinite-impulse-response (IIR) filter with a cut-off $(-3 \mathrm{~dB}$ point $)$ at $\frac{1}{3.3 \mathrm{~nm}}$ was applied to the extracted 2-D spectra to remove the low-frequency variability. This filter was optimized through repeated tests on synthetic $\mathrm{OH}$ spectra to have minimal impact on the temperatures derived from the $\mathrm{OH}$ rotational lines. Figure $3 \mathrm{a}$ and $\mathrm{b}$ show the $(5,3)$ vibrational band before and after application of this filter. After filtering, the spectrum was normalized such that the sum over the absolute values of the spectrum was equal to 1 .

A model spectrum was created using the $\mathrm{OH}$ line strengths, $S_{j^{\prime} j^{\prime \prime}}$, for a given vibrational transition, tabulated in the HIgh-resolution TRANsmission (HITRAN) molecular absorption database (Rothman et al., 2013). The number of photons emitted in each rotational transition, assuming a Boltzmann distribution of population in the upper-state rotational levels, is given by

$I=N_{v^{\prime}} \cdot S_{j^{\prime} j^{\prime \prime}} \cdot\left(v_{j^{\prime} j^{\prime \prime}}\right)^{3} \cdot \exp \left(-\frac{E_{j^{\prime}}}{k T_{\text {rot }}}\right)$.

Here $N_{v^{\prime}}$ is the relative population of the upper vibrational level, $v^{\prime}$, and $v_{j^{\prime} j^{\prime \prime}}$ is the wavenumber of the transition from the upper state level at potential energy $E_{j^{\prime}}$ to the final rotational state at energy $E_{j^{\prime \prime}}$. To form the model spectrum, these relative line intensities were convolved with the instrumental line shape and then filtered and normalized in the same manner as the data. A $\chi^{2}$ minimization using the rotational temperature and a total intensity scaling factor as the variable parameters was then performed between the model and the data to determine the rotational temperature best fitting the data. A Brent algorithm (Brent, 1973) from the GNU Scientific Library (Galassi and Gough, 2009) was used for the minimization. Following Pendleton et al. (1989), only rotational lines originating from levels $N \leq 4$ were used in the fitting, since higher levels may not be thermalized. The temperature fitting routine was tested against model data with added Gaussian white noise where temperatures were varied between 150 and $400 \mathrm{~K}$. The resulting fit was found to reproduce the input rotational temperatures to within the fitting errors of the temperature parameter.

Figure $3 \mathrm{c}$ shows the data from Fig. $3 \mathrm{~b}$ along with the fitted model spectrum shown as the red line. The fitted temperature in this case is found to be $186.5 \pm 0.7 \mathrm{~K}$, representing a $0.4 \%$ relative uncertainty. The residual spectrum (data fit) is shown in Fig. 3d, where it may be seen that, although small differences in the wavelength calibration or line shape may occur, the fitted spectrum accurately represents the observation. This and similar tests for the other Meinel bands and different integration times demonstrate that the model used is robust, that fitting the high-pass-filtered data works well, and that the fit to the data converges to a temperature with a small error.

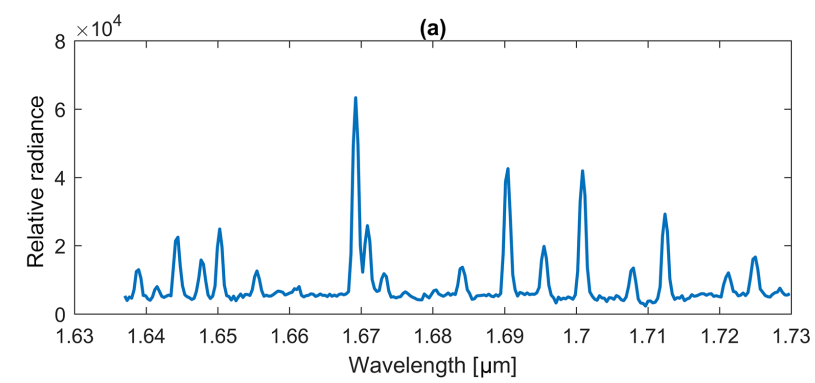

(b)

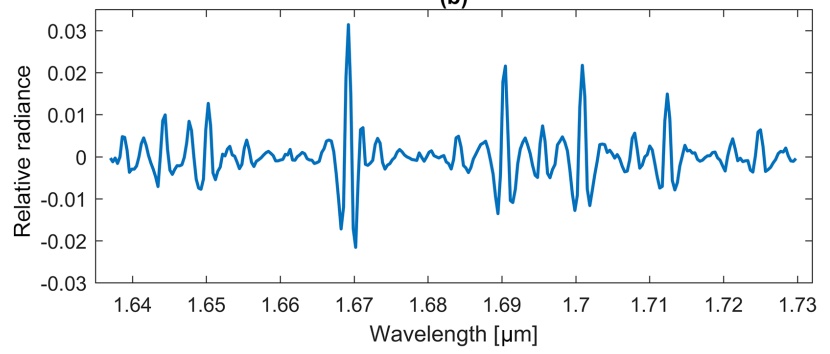

(c)

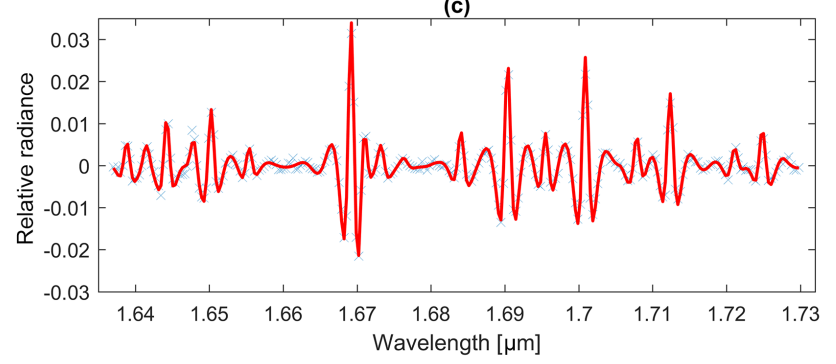

(d)

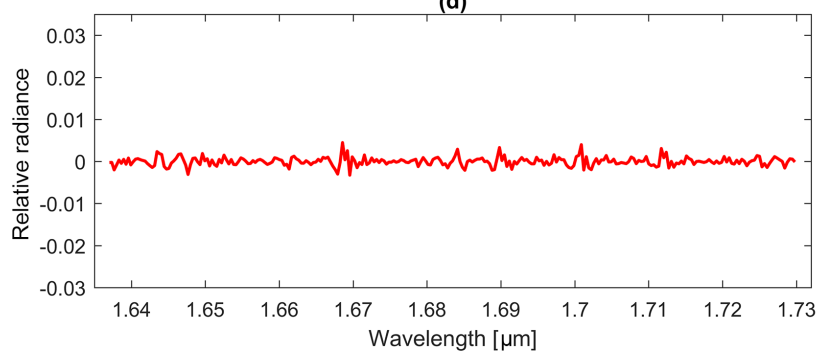

Figure 3. (a) Unfiltered spectrum of the $(5,3)$ transition. (b) Filtered spectrum of the $(5,3)$ transition. (c) Normalized spectrum from (b) (blue crosses) with the fitted, filtered model spectrum shown in red. The fitted rotational temperature is $186.5 \pm 0.7 \mathrm{~K}$. (d) Residuals of the fit from (c).

\subsection{Other vibrational bands}

Figure 4 shows an overview of all the measured $\mathrm{OH}$ vibrational transitions recorded in the $\mathrm{H}-, \mathrm{J}-$, and $\mathrm{K}$-band spectra taken toward the same star, extracted in the manner outlined above. The measurements in the $\mathrm{J}$ and $\mathrm{K}$ band were executed at 05:53 and 05:47 UT, respectively, meaning that the total time between all observations was about $6 \mathrm{~min}$. All spectra were taken with the same $10.8 \mathrm{~s}$ integration time and are normalized to the same Q-branch intensity for comparison. For the majority of vibrational transitions, the signal is 


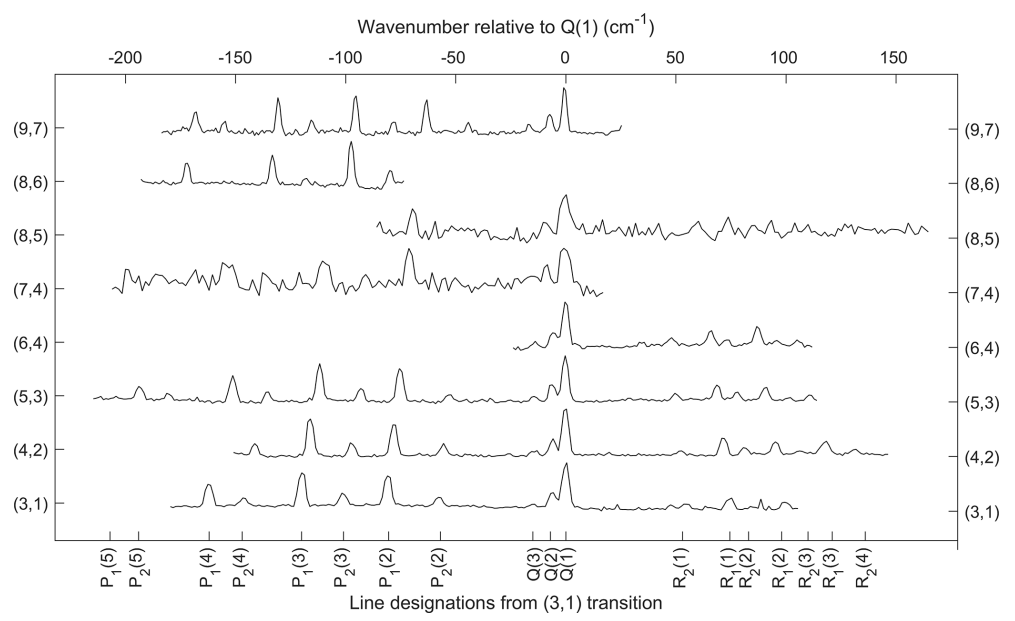

Figure 4. Spectra from the H, J, and $\mathrm{K}$ bands in wavenumbers relative to the $\mathrm{Q}(1)$ line in each band. The Q(1) lines of each spectrum are aligned. Line positions for the $(3,1)$ transition are shown.

very much greater than the noise, providing excellent data for temperature fitting, especially for the transitions in the $\mathrm{H}$ and $\mathrm{K}$ bands.

Since the $(7,4)$ and $(8,5)$ transitions in the $\mathrm{J}$ band have a $\Delta v=3$, the lines are not as intense as in the other $\Delta v=2$ bands. Due to the lower intensities of these $\Delta v=3$ bands, the signal-to-noise ratio is substantially reduced for this short integration, which is typical of the NOTCam data. As a result, the fitting error for these bands was very large. While longer exposures, or the co-addition of sequential short exposures, would improve the fitting error of the $\mathrm{OH}(7,4)$ band, the $\mathrm{R}$ and $\mathrm{Q}$ branches of the $(8,5)$ band are overlapped by the optically thick $\mathrm{O}_{2}$ infrared atmospheric band at $1270 \mathrm{~nm}$, and the filter cut-off reduces its $\mathrm{P}_{1}(4)$ line to near the noise level. Thus, the temperatures for the $(8,5)$ bands would remain compromised even for longer integrations.

\subsection{Temperature gradient}

The peak concentrations of the neighbouring vibrational levels are, on average, separated in altitude by $0.5 \mathrm{~km}$ (von Savigny et al., 2012). Even though the absolute peak altitudes are known to vary with season (Gao et al., 2010), they can be taken as constant on the timescales of a few minutes over which this experiment was executed. With a steady-state $\mathrm{OH}$ model driven using a neutral atmosphere from the Naval Research Laboratory Mass Spectrometer and Incoherent Scatter Radar Empirical model (NRLMSISE) (Picone et al., 2002), the altitude of the $(9,7)$ transition was fixed. The relative altitudes of the other vibrational transitions were then assigned using an altitude separation of $0.5 \mathrm{~km}$, the average separation found by von Savigny et al. (2012). The rotational temperatures derived from each of the individual vibrational bands then provide an estimate of temperature gradients present similar to what has been done by Perminov et al. (2007) and Schubert et al. (1990).

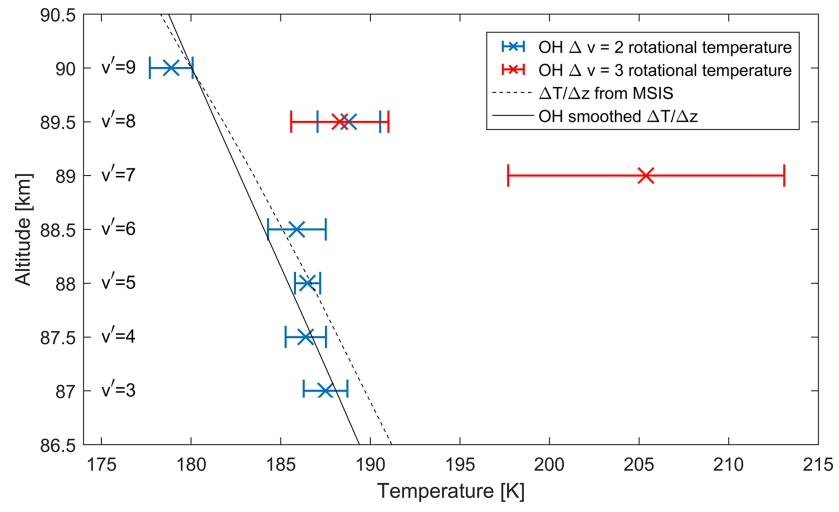

Figure 5. The temperatures from the data presented in Fig. 4 with $\Delta v=2$ in blue and $\Delta v=3$ in red. Both are presented with their $1 \sigma$ error bar. The altitude of the $(9,7)$ transition was fixed with a steadystate model using a neutral atmosphere. The altitudes of the other transitions are fixed with a relative distance between neighbouring transitions of $0.5 \mathrm{~km}$, following von Savigny et al. (2012). The NRLMSISE temperature gradient for 19 February 2013 at La Palma $\left(17^{\circ} 53^{\prime} \mathrm{W}, 28^{\circ} 45^{\prime} \mathrm{N}\right)$ at 05:50 UT is shown as a dashed black line. The same gradient but smoothed with a modelled $\mathrm{OH}$ distribution in altitude is shown as a solid black line.

Figure 5 shows the distribution in altitude for the data presented here together with the NRLMSISE model kinetic temperature for the corresponding location and time. In addition, the NRLMSISE profile has been smoothed with the volume emission rate profile of an $\mathrm{OH}$ band derived from the steadystate $\mathrm{OH}$ model to reflect the effect of the $\mathrm{OH}$ layer width on the rotational temperatures. The temperature profile from this model is consistent with the gradients estimated from our data for the $(3,1),(4,2),(5,3),(6,4)$, and $(9,7)$ transitions. It is important to note that the $\mathrm{J}$-, $\mathrm{H}$-, and $\mathrm{K}$-band data shown in Fig. 5 were acquired using sequential $10.8 \mathrm{~s}$ observations, spanning only $6 \mathrm{~min}$. Thus, large deviations from the 
climatological gradients can occur due to wave activity $(\mathrm{Xu}$ et al., 2000). While the agreement here may be fortuitous, a time sequence of short integrations may give insight into the wave-induced temperature gradients.

While the $(8,6)$ transition is anomalously high, it was found that the $\mathrm{P}(2)$ and $\mathrm{P}(4)$ lines are partially absorbed by atmospheric $\mathrm{H}_{2} \mathrm{O}$ and $\mathrm{CO}_{2}$ (Jones et al., 2013; Noll et al., 2012). To model the impact of this atmospheric absorption, synthetic spectra with rotational temperatures between 130 and $300 \mathrm{~K}$ were created. When these were fitted with the technique described above, this input temperature could be retrieved. When these synthetic spectra were however first multiplied by a high-resolution $(0.002 \mathrm{~nm})$ absorption spectrum for seasonally averaged conditions obtained from the Cerro Paranal sky model (Jones et al., 2013; Noll et al., 2012), the fitted temperature was approximately $8 \%$ higher than the original synthesized temperature. This would account for the higher fitted temperature of the observed $(8,6)$ band shown in Fig. 5. Using this same technique, the temperatures for all other vibrational-rotational transitions presented in Fig. 5 were examined and found not to be significantly affected by atmospheric absorption.

With the intent of demonstrating that the NOTCam provides atmospheric data that can be used to supplement other astronomical data sets used for aeronomic studies (e.g. Osterbrock et al., 1996; Noll et al., 2015; Cosby and Slanger, 2007), a sample short-integration spectrum was analysed, and both the analysis procedure and the results are presented. With the exception of the weak or compromised $v^{\prime}=7$ and 8 levels discussed earlier, the temperature variation with vibrational level observed using the NOTCam and analysed here reflects the NRLMSISE kinetic temperature to within $2 \sigma$. This is consistent with previous observations using ground-based spectrometers or interferometers (e.g. Innis et al., 2001; Oberheide et al., 2006; French and Mulligan, 2010; Dyrland et al., 2010). However, it must be pointed out that the NOTCam measurements cover a very short time span and may be affected by gravity-wave perturbations of the climatological temperature gradient of the atmosphere represented by NRLMSISE. The temperature distribution with vibrational level observed here shows a small decrease toward higher levels, although this decrease is, apart from the $v^{\prime}=9$, not significant at the $2 \sigma$ level. This is at odds with the measurements of Noll et al. (2015) and Cosby and Slanger (2007), who show strong increases in temperature with vibrational level that they attribute to nonthermodynamic equilibrium effects. However, our results are consistent with the results of Lübken et al. (1990), Espy and Hammond (1995), Wrasse et al. (2004), and Perminov et al. (2007), who show similar or decreasing temperatures with increasing vibrational level. Once again, the short duration of these demonstration data is not able to resolve this apparent discrepancy, but the larger NOTCam data set may prove useful in this regard.

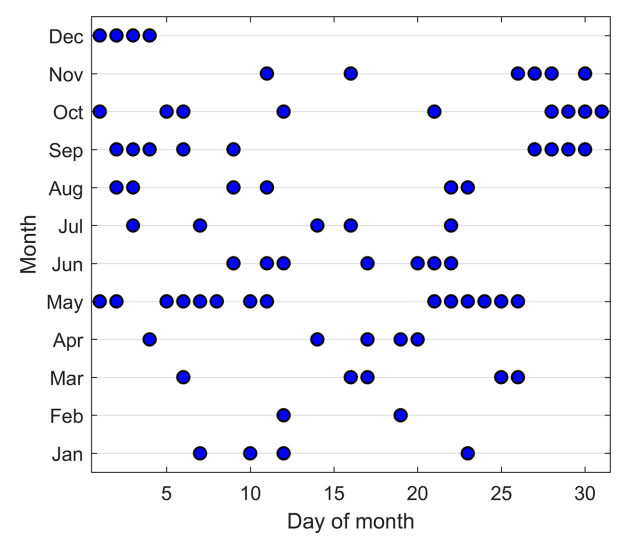

Figure 6. Dates between November 2007 and June 2016 (which is the coverage in the NOTCam archive) when the NOTCam was mounted at the NOT and making spectroscopic astronomical observations.

\section{Outlook}

Near-IR spectroscopy with NOTCam has been performed over the last 13 years toward point-like sources (stars or marginally extended objects). NOTCam, in both spectroscopic and imaging mode, was mounted and used on the telescope an average of $15 \%$ of the observing time between 2003 and 2016, spread evenly throughout the calendar year. Thus, good seasonal coverage is available as indicated in Fig. 6, which shows the distribution of dates between November 2007 and June 2016 when the NOTCam was used for long-slit spectroscopic observations.

The spectroscopic mode has been continuous and stable with no change of optical elements, except for the addition of the two broadband filters, $Z$ and $Y$, in 2010. There has been a change of detector, most recently in 2007, but each array is well characterized. All data older than 1 year (the proprietary period at the NOT) are available in the NOT data archive. Figure 7 shows histograms of the total number of spectroscopic observations taken in the $\mathrm{J}, \mathrm{H}$, and $\mathrm{K}$ bands for each hour, indicating nearly uniform coverage in each wavelength region.

With this data set, a variety of atmospheric problems can be addressed. One example is to simply generate climatological temperature gradients of the mesopause region from the long-term data as in Fig. 5. High-quality long-term observations of the mean state, trends, and inter-annual variability of this region are rare, especially at low latitudes, and can serve as an important standard against which whole atmosphere models can be validated.

Additionally, there is a long-standing discussion as to whether the vibrationally excited $\mathrm{OH}$ quenches to the ground state ("sudden death") or relaxes to the next-lowest vibrational level (McDade, 1991). Knowledge of the population and quenching of the individual upper states is essential for the interpretation of the $\mathrm{OH}$ airglow emission for remote 

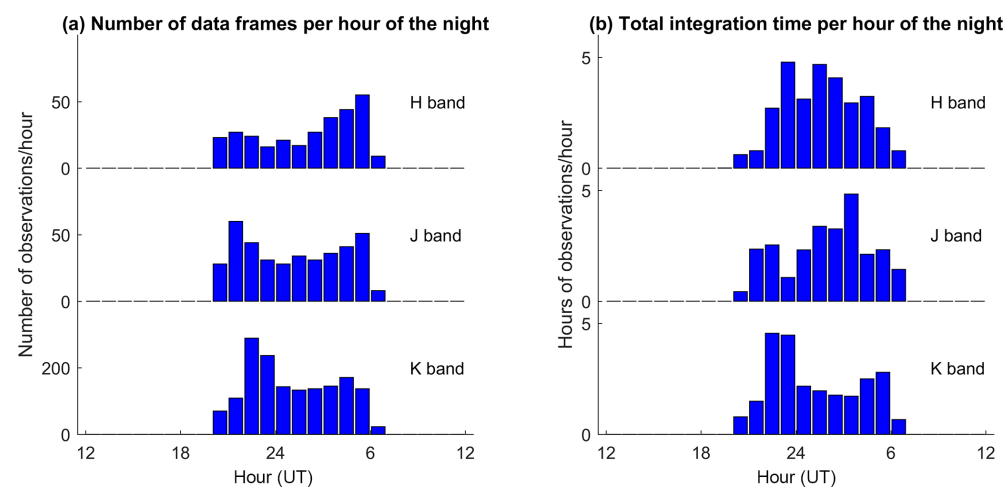

Figure 7. (a) Total number of spectroscopic data frames recorded in each hour and each band with NOTCam between November 2007 and June 2016. (b) Total hours of on-target integration time for spectroscopic data recorded in each hour and each band with the NOTCam in the same period of time.

sensing of the mesopause region. These high-quality data described above, together with a steady-state model, can be used to estimate the ratio of single to multi-quantum quenching efficiency accurately. Although these quenching rates have been examined by Xu et al. (2012) using broadband SABER measurements that combine multiple high- $v^{\prime}$ and low- $v^{\prime}$ levels, the individual bands resolved by astronomical telescopes would allow an unambiguous assessment of these rates.

The data discussed here have very short integration times as a demonstration of the worst-case conditions. This means that a series of measurements of the same spot of the sky can scan the $\mathrm{OH}$ layer with a repetition rate of down to $20 \mathrm{~s}$. This lies well below the upper boundary of gravity waves (Kovalam et al., 2011) and stretches into the domain of acoustic and infrasonic waves. Although these waves have been observed in the hydroxyl airglow (Bittner et al., 2010; Pilger et al., 2013), the low spatial resolution of the observations creates ambiguity in their identification. Here the observations have a spatial resolution on the airglow layer of about $100 \mathrm{~m}$ (which is 4 arcmin), sampled with approximately 1000 pixels in the spatial direction of the NOT detector. This resolution is achieved with the telescope in "staring mode", which means without star tracking. The resolution with star tracking is dependent on the slit orientation and is in the worst case up to $6.7 \times 2.7$ arcmin over a $10.8 \mathrm{~s}$ integration. Using staring mode, the high resolution in both the temporal and spatial dimensions allows high-frequency waves to be measured at a commensurate spatial resolution. This opens up new possibilities to study the smallest structures of waves propagating through the $\mathrm{OH}$ layer in the MLT. While staring observations would recover the full spatial resolution of the instrument, care should be taken with the slit orientation and choice of direction to ensure that bright astronomical objects do not interfere with the airglow observations.

Finally, in parallel with the atmospheric work, it will be possible to quantify how the intensity of the mesospheric $\mathrm{OH}$ background in the astronomical $\mathrm{H}, \mathrm{J}$, and $\mathrm{K}$ bands varies on different timescales (from minutes to years) over La Palma. This will be useful in the planning and scheduling of observations in order to optimize dithering strategies and observing modes, especially important for instruments observing simultaneously at optical and infrared wavelengths.

Data availability. Archive data from the NOTCam can be accessed via http://www.not.iac.es/observing/forms/fitsarchive/ (Nordic Optical Telescope, 2017).

Competing interests. The authors declare that they have no conflict of interest.

Acknowledgements. This work was based on observations made with the Nordic Optical Telescope, operated by the Nordic Optical Telescope Scientific Association at the Observatorio del Roque de los Muchachos, La Palma, Spain, of the Instituto de Astrofisica de Canarias. We thank the staff from the NOT for their help and support during a visit by Christoph Franzen in November 2014. This work was supported by the Research Council of Norway/Centres of Excellence (CoE) under contract 223252/F50.

Edited by: Lars Hoffmann

Reviewed by: two anonymous referees

\section{References}

Abbott, T. M., Aspin, C., Sorensen, A. N., Norregaard, P., Andersen, J., Andersen, M. I., van der Bliek, N. S., Clasen, J. W., Cox, G. C., Klougart, J., Larsen, H. H., Michaelsen, N., Noel, B., Olofsson, G., Perez, C., and Schwarz, H. E.: SWIR at the Nordic Optical Telescope: NOTCam, in: Optical and IR Telescope Instrumentation and Detectors, Vol. 4008, edited by: Iye, M. and Moorwood, A. F., SPIE Proc., Munich, 2000. 
Baker, D. J. and Stair Jr., A. T.: Rocket measurements of the altitude distributions of the hydroxyl airglow, Phys. Scripta, 37, 611-622, 1988.

Bittner, M., Höppner, K., Pilger, C., and Schmidt, C.: Mesopause temperature perturbations caused by infrasonic waves as a potential indicator for the detection of tsunamis and other geo-hazards, Nat. Hazards Earth Syst. Sci., 10, 1431-1442, https://doi.org/10.5194/nhess-10-1431-2010, 2010.

Brent, R. P.: Algorithms for Minimization Without Derivatives, Dover Publications, Mineola, NY, 1973.

Cosby, P. C. and Slanger, T. G.: OH spectroscopy and chemistry investigated with astronomical sky spectra, Can. J. Phys., 85, 7799, 2007.

Djupvik, A. A.: http://www.not.iac.es/instruments/notcam/, last access: 10 August 2016.

Djupvik, A. A. and Andersen, J.: The Nordic Optical Telescope, in: Highlights of Spanish Astrophysics V, edited by: Diego, M. J., Goicoechea, J. L., González-Serrano, I. J., and Gorgas, J., Springer, Berlin, Heidelberg, 2010.

Dyrland, M. E., Mulligan, F. J., Hall, C. M., Sigernes, F., Tsutsumi, M., and Deehr, C. S.: Response of $\mathrm{OH}$ airglow temperatures to neutral air dynamics at $78^{\circ} \mathrm{N}, 16^{\circ}$ E during the anomalous 20032004 winter, J. Geophys. Res.-Atmos., 115, 1-11, 2010.

Espy, P. J. and Hammond, M. R.: Atmospheric transmission coefficients for hydroxyl rotational lines used in rotational temperature determinations, J. Quant. Spectrosc. Ra., 54, 879-889, 1995.

Espy, P. J. and Stegman, J.: Trends and variability of mesospheric temperature at high-latitudes, Phys. Chem. Earth, 27, 543-553, 2002.

French, W. J. R. and Mulligan, F. J.: Stability of temperatures from TIMED/SABER v1.07 (2002-2009) and Aura/MLS v2.2 (2004-2009) compared with OH(6-2) temperatures observed at Davis Station, Antarctica, Atmos. Chem. Phys., 10, 1143911446, https://doi.org/10.5194/acp-10-11439-2010, 2010.

Galassi, M. and Gough, B.: GNU Scientific Library Reference Manual, 3rd Edn., Network Theory Ltd, Godalming, UK, 2009.

Gao, H., Xu, J., and Wu, Q.: Seasonal and QBO variations in the $\mathrm{OH}$ nightglow emission observed by TIMED/SABER, J. Geophys. Res.-Space, 115, 1-13, 2010.

Innis, J. L., Phillips, F. A., Burns, G. B., Greet, P. A., French, W. J. R., and Dyson, P. L.: Mesospheric temperatures from observations of the hydroxyl (6-2) emission above Davis, Antarctica: A comparison of rotational and Doppler measurements, Ann. Geophys., 19, 359-365, https://doi.org/10.5194/angeo-19-359-2001, 2001.

Jones, A., Noll, S., Kausch, W., Szyszka, C., and Kimeswenger, S.: An advanced scattered moonlight model for Cerro Paranal, Astron. Astrophys., 560, 1-11, 2013.

Kovalam, S., Tsuda, T., and Gurubaran, S.: High-frequency gravity waves observed in the low-latitude mesosphere-lower thermosphere (MLT) region and their possible relationship to loweratmospheric convection, J. Geophys. Res.-Atmos., 116, D15101, https://doi.org/10.1029/2011JD015625, 2011.

Lübken, F. J., von Zahn, U., Manson, A., Meek, C., Hoppe, U. P., Schmidlin, F. J., Stegman, J., Murtagh, D. P., Rüster, R., Schmidt, G., Widdel, H. U., and Espy, P.: Mean state densities, temperatures and winds during the MAC/SINE and MAC/EPSILON campaigns, J. Atmos. Terr. Phys., 52, 955-970, 1990.
McDade, I. C.: The altitude dependence of the $\mathrm{OH}(\mathrm{X} 2 \Pi)$ vibrational distribution in the nightglow: Some model expectations, Planet. Space Sci., 39, 1049-1057, 1991.

Meinel, I. A. B.: OH Emission Bands in the Spectrum of the Night Sky. II, Astrophys. J., 112, 120-130, 1950.

Noll, S., Kausch, W., Barden, M., Jones, A. M., Szyszka, C., Kimeswenger, S., and Vinther, J.: An atmospheric radiation model for Cerro Paranal, Astron. Astrophys., 543, A92, 2012.

Noll, S., Kausch, W., Kimeswenger, S., Unterguggenberger, S., and Jones, A. M.: $\mathrm{OH}$ populations and temperatures from simultaneous spectroscopic observations of 25 bands, Atmos. Chem. Phys., 15, 3647-3669, https://doi.org/10.5194/acp-153647-2015, 2015.

Nordic Optical Telescope: The NOT FITS Header Archive, available at: http://www.not.iac.es/observing/forms/fitsarchive/, last access: 22 August 2017.

Oberheide, J., Offermann, D., Russell, J. M., and Mlynczak, M. G.: Intercomparison of kinetic temperature from $15 \mu \mathrm{m} \mathrm{CO}_{2} \operatorname{limb}$ emissions and $\mathrm{OH}^{*}(3,1)$ rotational temperature in nearly coincident air masses: SABER, GRIPS, Geophys. Res. Lett., 33, 1-5, 2006.

Osterbrock, D. E., Fulbright, J. P., Martel, A. R., Keane, M. J., Trager, S. C., and Basri, G.: Night-Sky High-Resolution Spectral Atlas of $\mathrm{OH}$ and $\mathrm{O}_{2}$ Emission Lines for Echelle Spectrograph Wavelength Calibration, Publ. Astron. Soc. Pacific, 108, 277-308, 1996.

Pendleton, W. R., Espy, P. J., Baker, D. J., Steed, A., Fetrow, M. P., and Henriksen, E. K.: Observation of $\mathrm{OH}$ Meinel $(7,4)$ $P\left(N^{\prime \prime}=13\right)$ transitions in the night airglow, J. Geophys. Res.Space, 94, 505-510, 1989.

Pendleton, W. R., Espy, P. J., and Hammond, M. R.: Evidence for non-local-thermodynamic-equilibrium in the $\mathrm{OH}$ nightglow, J. Geophys. Res., 98, 11567-11579, 1993.

Perminov, V. I., Semenov, A. I., and Shefov, N. N.: On rotational temperature of the hydroxyl emission, Geomagn. Aeron., 47, 756-763, 2007.

Picone, J. M., Hedin, A. E., Drob, D. P., and Aikin, A. C.: NRLMSISE-00 empirical model of the atmosphere: Statistical comparisons and scientific issues, J. Geophys. Res.-Space, 107, 1468, https://doi.org/10.1029/2002JA009430, 2002.

Pilger, C., Schmidt, C., Streicher, F., Wüst, S., and Bittner, M.: Airglow observations of orographic, volcanic and meteorological infrasound signatures, J. Atmos. Sol.-Terr. Phy., 104, 55-66, 2013.

Rothman, L. S., Gordon, I. E., Babikov, Y., Barbe, A., Chris Benner, D., Bernath, P. F., Birk, M., Bizzocchi, L., Boudon, V., Brown, L. R., Campargue, A., Chance, K., Cohen, E. A., Coudert, L. H., Devi, V. M., Drouin, B. J., Fayt, A., Flaud, J. M., Gamache, R. R., Harrison, J. J., Hartmann, J. M., Hill, C., Hodges, J. T., Jacquemart, D., Jolly, A., Lamouroux, J., Le Roy, R. J., Li, G., Long, D. A., Lyulin, O. M., Mackie, C. J., Massie, S. T., Mikhailenko, S., Müller, H. S. P., Naumenko, O. V., Nikitin, A. V., Orphal, J., Perevalov, V., Perrin, A., Polovtseva, E. R., Richard, C., Smith, M. A. H., Starikova, E., Sung, K., Tashkun, S., Tennyson, J., Toon, G. C., Tyuterev, V. G., and Wagner, G.: The HITRAN2012 molecular spectroscopic database, J. Quant. Spectrosc. Ra., 130, 4-50, 2013.

Schubert, G., Walterscheid, R. L., Hecht, J. H., and Sivjee, G. G.: Temperature gradients at mesopause heights inferred from $\mathrm{OH}$ nightglow data, J. Geophys. Res.-Space, 95, 19061-19067, 1990. 
Sigernes, F., Shumilov, N., Deehr, C. S., Nielsen, K. P., Svenøe, T., and Havnes, O.: Hydroxyl rotational temperature record from the auroral station in Adventdalen, Svalbard, J. Geophys. Res.Space, 108, 1342-1351, 2003.

Smith, A. K.: Global Dynamics of the MLT, Surv. Geophys., 33, 1177-1230, 2012.

Smith, A. K.: Physics and chemistry of the mesopause region, J. Atmos. Sol.-Terr. Phy., 66, 839-857, 2004.

Smith, S. M., Baumgardner, J., Mertens, C. J., Russell, J. M., Mlynczak, M. G., and Mendillo, M.: Mesospheric OH temperatures: Simultaneous ground-based and SABER OH measurements over Millstone Hill, Adv. Space Res., 45, 239-246, 2010.

Telting, J.: http://www.not.iac.es/instruments/notcam/ spectroscopy/, last access: 10 August 2016.

Tody, D.: IRAF in the Nineties, Astronomical Data Analysis Software and Systems II, A. S. P. Conference Series, San Francisco, USA, 52, 173, 1993.

von Savigny, C., McDade, I. C., Eichmann, K.-U., and Burrows, J. P.: On the dependence of the $\mathrm{OH}^{*}$ Meinel emission altitude on vibrational level: SCIAMACHY observations and model simulations, Atmos. Chem. Phys., 12, 8813-8828, https://doi.org/10.5194/acp-12-8813-2012, 2012. von Zahn, U., Fricke, K. H., Gerndt, R., and Blix, T.: Mesospheric temperatures and the $\mathrm{OH}$ layer height as derived from groundbased lidar and $\mathrm{OH}^{*}$ spectrometry, J. Atmos. Terr. Phys., 49, 863-869, 1987.

Wrasse, C. M., Takahashi, H., and Gobbi, D.: Comparison of the $\mathrm{OH}(8-3)$ and (6-2) band rotational temperature of the mesospheric airglow emissions, Rev. Bras. Geofis., 22, 223-231, 2004.

Xu, J., Gao, H., Smith, A. K., and Zhu, Y.: Using TIMED/SABER nightglow observations to investigate hydroxyl emission mechanisms in the mesopause region, J. Geophys. Res.-Atmos., 117, $1-22,2012$.

Xu, J., Smith, A. K., and Brasseur, G. P.: The effects of gravity waves on distributions of chemically active constituents in the mesopause region, J. Geophys. Res.-Atmos., 105, 26593-26602, 2000.

Yee, J.-H., Crowley, G., Roble, R. G., Skinner, W. R., Burrage, M. D., and Hays, P. B.: Global simulations and observations of $\mathrm{O}(1 \mathrm{~S}), \mathrm{O}_{2}$ (1Sigma) and $\mathrm{OH}$ mesospheric nightglow emissions, J. Geophys. Res.-Space, 102, 19949-19968, 1997. 